\title{
Lymphomatosis cerebri : un défi diagnostic clinique et radiologique
}

\section{Lymphomatosis cerebri: a clinical and radiological diagnosis challenge}

E Berling ${ }^{1,6}$, Dr A-C Zeghoudi ${ }^{1}$, Dr J Servan ${ }^{1}$, Dr J Yeung ${ }^{1,6}$, Dr S Aldea ${ }^{2}$, Dr M De Malherbe $^{3}$, Dr F Bielle ${ }^{4}$ Dr J-P Bedos ${ }^{5}$, Dr S Legriel ${ }^{5}$, Pr F Pico ${ }^{1,6}$

1 Service de neurologie, Centre hospitalier de Versailles, France

2 Service de neurochirurgie, Hôpital Foch, Suresnes, France

3 Service de radiologie, Centre hospitalier de Versailles, France

4 Service de neuropathologie, Hôpital de la Pitié-Salpêtrière, APHP, Paris, France

5 Service de réanimation médico-chirurgicale, Centre hospitalier de Versailles, France

6 Université Versailles Saint Quentin en Yvelines et Paris Saclay, France

Correspondance : Edouard Berling, Centre Hospitalier de Versailles, Hôpital A. Mignot, 177 rue Versailles.78150 Le Chesnay. Tél : 07700652 47, E-mail : edouard.berling@ outlook.fr 


\section{RESUME :}

Nous rapportons le cas d'un homme de 60 ans, immunocompétent, présentant un tableau de méningo-encéphalite d'évolution subaiguë avec atteinte des noyaux gris centraux, du mésencéphale et de la substance blanche. La biopsie cérébrale n'était initialement pas réalisable. Après plusieurs semaines, d'évolution clinique et radiologique défavorable malgré une corticothérapie d'épreuve, de nouvelles lésions apparurent et un examen anatomopathologique permis le diagnostic de lymphomatose cérébrale. La lymphomatose cérébrale est une forme rare et diffuse de lymphome cérébral primitif avec une présentation radiologique différente de la présentation classique du lymphome cérébral primitif. Du fait de sa rareté et de sa présentation inhabituelle, la lymphomatose cérébrale est de diagnostic difficile avec de nombreux diagnostics différentiels.

Mots-clés : Lymphomatose cérébrale; Lymphome cérébral primitif

\section{ABSTRACT:}

We report the case of a 60 -year-old man with subacute meningoencephalitis involving lesions of the basal ganglia, the cerebral peduncles and white matter. Brain biopsy was initially not feasible. Steroid treatment was given but the course was unfavorable and new lesions appeared several weeks later. Brain biopsy with pathological examination allows the diagnosis of lymphomatosis cerebri. Lymphomatosis cerebri is a rare and diffuse form of primary cerebral lymphoma of difficult diagnosis due to its clinico-radiological presentation with several differential diagnoses

Keywords : Lymphomatosis cerebri; Primary central nervous system lymphoma 
Un homme âgé de 60 ans fut admis en août 2016 pour ataxie et somnolence évoluant depuis un mois, avec comme antécédents une intoxication alcoolo-tabagique, une hypertension artérielle primitive, une artérite oblitérante des membres inférieurs ainsi qu'une endartériectomie de la carotide interne droite. Son traitement comprenait du clopidogrel, une statine, une trithérapie anti-hypertensive (lercanidipine, hydrochlorothiazide, enalapril).

Le patient se plaignait d'une augmentation du temps de sommeil et de céphalées pariétales droites à prédominance matinales. L'examen clinique objectiva un ralentissement idéique, un syndrome dysexécutif sévère, et un syndrome cérébelleux gauche. Le reste du bilan neurologique et général était sans particularité.

Une première IRM cérébrale (fig. 1 et 2) retrouva des hypersignaux FLAIR bipallidaux, bithalamiques, du bras postérieur de la capsule interne, du mésencéphale étendus au pédoncule cérébelleux moyen gauche ainsi qu'un hypersignal cortico-sous-cortical frontal droit, sans effet de masse, ni prise de contraste. La diffusion mis en évidence des hypersignaux dans la partie antérieure du mésencéphale, sans restriction de l'ADC. La spectroscopie sur le pallidum droit était sans anomalie, ainsi que la perfusion. A noter, la présence de quelques lacunes et d'une leucopathie vasculaire déjà connues.

L'étude du liquide cérébro-spinal révéla une méningite avec 21 éléments dont $97 \%$ de lymphocytes T matures polyclonaux, avec protéinorachie à $0.37 \mathrm{~g} / \mathrm{l}$ sans bandes oligoclonales, et une normoglycorachie. Les cultures, incluant Mycobacterium tuberculosis, les PCR virales et bactériennes (HSV, VZV, EBV, entérovirus, Mycobacterium tuberculosis, Tropheryma whipplei), les sérologies (Borrelia burgdorferi, Toxoplasma gondii), l'antigène cryptococcique, furent négatifs, ainsi que la protéine 14-3-3. Un rapport interleukine-10 /interleukine-6 inversé à 3.3 (norme inférieure à 1) fut retrouvé.

L'étude du sérum ne retrouva ni syndrome inflammatoire biologique, ni anomalie ionique, métabolique ou carentielle. Un bilan infectieux (hémocultures, sérologie Syphilis, VIH, VHB, VHC, PCR Whipple dans la salive et les selles) ainsi qu'un bilan auto-immun (ANCA, FAN, anticorps anti-ADN natifs, anti TPO, anti-TG, anti-aquaporine 4, anti MOG, anti-neuronaux cellulaireset membranaires, l'enzyme de conversion de l'angiotensine, la recherche HLA B51) revinrent négatifs.

Une TEP-TDM, une échographie testiculaire et un examen ophtalmologique ne mirent en évidence aucune anomalie. Un électro-encéphalogramme ne retrouva qu'un ralentissement du rythme de fond avec quelques surcharges lentes prédominant en temporal droit.

Après élimination des étiologies infectieuses, métaboliques, carentielles et systémiques, deux hypothèses furent discutées : lymphome cérébral primitif et encéphalite auto-immune ou 
paranéoplasique. Cette dernière n'étant étayée ni par un tableau clinique classique, ni par la présence d'une tumeur ou d'un anticorps spécifique. Après discussion avec les neurochirurgiens, l'impraticabilité d'une biopsie fut retenue en raison de la localisation profonde des lésions.

Deux semaines après l'admission, l'état clinique du patient se dégrada avec l'aggravation du syndrome dysexécutif, l'apparition de troubles de la vigilance, puis la survenue d'un état de mal épileptique généralisé nécessitant son transfert en réanimation.

Compte-tenu de l'inaccessibilité des lésions à une éventuelle biopsie et de la gravité du tableau clinique, une corticothérapie d'épreuve fut débutée par méthylprednisolone $2 \mathrm{~g}$ intraveineux en 2 jours puis $3 \mathrm{~g}$ en 3 jours et enfin suivie, 2 semaines après, de $60 \mathrm{mg} / \mathrm{j}$ de prednisone per os. Devant la poursuite de l'aggravation clinique et radiologique du patient présentant un Glasgow Coma Scale à 3, $1 \mathrm{~g}$ de rituximab intraveineux fut également délivré, sans amélioration.

A 1 mois du début de la corticothérapie et 2 semaines environ du rituximab, avec la répétition des explorations, une nouvelle IRM cérébrale (fig.3) fit apparaître, en séquence FLAIR, une importante majoration symétrique des hypersignaux diencéphaliques avec extension temporomésiale, pariéto-frontale et cérébelleuse. Après l'injection de gadolinium, les lésions prenaient le contraste en plages, de façon homogène, intense, sans effet de masse. Il existait une restriction sur la cartographie ADC.

Devant ces lésions évocatrices de lymphome cérébral primitif et leur accessibilité, une biopsie stéréotaxique fut réalisée dans l'hémisphère cérébelleux gauche. L'examen anatomopathologique (fig.4) retrouva une infiltration du tissu nerveux par de rares grandes cellules lymphoïdes CD20+ et CD79+ isolées les unes des autres. Le diagnostic de lymphomatose cérébrale fut alors évoqué, malgré le faible nombre de cellules lymphoïdes probablement expliquée par les thérapies précédemment entreprises, notamment la corticothérapie.

Par la suite, un traitement par 1 cure de méthotrexate IV $\left(3 \mathrm{~g} / \mathrm{m}^{2}\right)$ fut instauré avec dans les suites une disparition totale des prises de contrastes. Cette réponse incontestable confirma le diagnostic. Malheureusement sur l'IRM de suivi (fig.5), sont apparus des hypersignaux T1, intenses, des deux noyaux caudés et des putamens, non superposables aux prises de contraste signant une nécrose hémorragique dont l'étiologie resta indéterminée bien qu'une toxicité du méthotrexate fut suspectée. Une décision d'arrêt des thérapeutiques actives fut prise de manière collégiale en respectant la législation en vigueur. Le patient décéda au décours immédiat de l'arrêt de la ventilation artificielle. 


\section{Commentaires}

Notre cas illustre la difficulté diagnostique de la lymphomatose cérébrale, qui, du fait de sa rareté, est mal connue. Notre patient, immunocompétent, présentait un tableau aspécifique d'encéphalopathie subaigüe sans atteinte extra-neurologique avec une IRM n'évoquant pas en première intention un processus tumoral du fait de ses anomalies de signal exclusivement en séquence T2 et FLAIR, de la symétrie des lésions, de l'absence de prise de contraste, de la normalité des séquences d'ADC et de perfusion ainsi que de la spectroscopie. Les nombreux diagnostics différentiels (infectieux, dysimmunitaires, carentielles et paranéoplasique) furent écartés par le bilan large biologique d'abord sur le sérum et le LCS du patient strictement négatif mais aussi par une TEP-TDM normale. L'élément orientant au départ vers un lymphome cérébral fut l'élévation dans le LCS de l'IL10 avec inversion du rapport IL10/IL6. Plusieurs mois après le début des symptômes, alors qu'un traitement d'épreuve par corticothérapie et rituximab avait échoué, une nouvelle IRM mit en évidence de nouvelles lésions ayant l'aspect plus habituel de celles des lymphomes cérébraux et rendant possible une biopsie. L'examen anatomopathologique et la réponse au traitement par méthotrexate confirma la suspicion radiologique de lymphome cérébral primitif sous sa forme de lymphomatose cérébrale. Malgré la réponse radiologique au traitement instauré, le patient s'aggrava justifiant la fin de toute thérapeutique invasive entrainant le décès du patient. L'étiologie de la nécrose striatale que présenta le patient demeura indéterminée, bien qu'une toxicité du méthotrexate fût fortement suspectée celle-ci n'est pas décrite dans la littérature ${ }^{1}$. La lymphomatose cérébrale est une forme rare de lymphome cérébral primitif qui est caractérisée par une infiltration diffuse de cellules lymphomateuses et non une masse cohésive de celles-ci. D’un point de vue épidémiologique, celle-ci semble toucher la même population que le lymphome cérébral primitif de l'immunocompétent, c'est-à-dire majoritairement des individus entre 50 et 70 ans, mais peut apparaitre à tout âge ${ }^{2}$, avec un sex-ratio de $1^{3}$. Dans la revue de la littérature de C. Izquierdo et al. ${ }^{3}$ faite sur 42 patients, la clinique est aspécifique mais il s'agit le plus souvent d'une démence rapidement progressive, dans 60 \% des cas, avec des symptômes neurologiques aussi variés que des troubles de la personnalité, du comportement ou de la mémoire, des crises d'épilepsie, des troubles de la

marche mais aussi une altération de l'état général avec une perte de poids fréquente ${ }^{4,5}$.A l'examen clinique, un déficit moteur focal est retrouvé dans environ 1 cas sur 3. Les uvéites 
semblent plus rares que dans les formes classiques de lymphome cérébral primitif, environ $5 \%^{3}$ contre $13 \%^{6}$.

L'imagerie classique à l'IRM est celle d'hypersignaux T2 et FLAIR diffus, souvent symétriques, à la fois supra et infra-tentoriels dans 55\% dans cas, et qui concernent quasiment toujours les 2 hémisphères ${ }^{7}$. A noter que, contrairement à la forme classique du lymphome cérébral primitif, les noyaux gris centraux, le tronc cérébral voire la moelle épinière ${ }^{8}$ sont très souvent impliqués. Classiquement, ces lésions ne présentent pas de prise de contraste ${ }^{9}$ ou une prise de contraste diffuse et discrète dans $1 / 3$ des cas. Cependant, sont décrites des lésions avec prise de contraste nodulaire, apparaissant plus volontiers au cours de l'évolution qu'initialement, comme ici. Nous manquons de cas rapportés pour définir le profil spectroscopique, cependant un pic de choline était retrouvé chez 4 des 5 patients ayant bénéficiés d'une spectroscopie dans la revue de C. Izquierdo et al., bien que ce ne fût pas le cas chez notre patient. Dans la même revue, une discrète restriction de l'ADC était rapportée dans 6 cas sur les 9 où celle-ci avait été recherchée, contrairement à notre patient initialement. Le LCS est le plus souvent anormal avec une pléiocytose modérée constituée de lymphocytes matures dans plus de la moitié des cas et une hyperprotéinorachie modérée dans $75 \%$ des cas. Chez $75 \%$ des patients, aucune cellule lymphomateuse n'est retrouvée, ce taux semble réduit en cas de recherche par cytométrie de flux ${ }^{3}$, qui doit donc être systématique en cas de forte suspicion. Dans notre cas, le taux d'IL-10 était élevé, ce dosage a démontré son utilité diagnostique dans le lymphome cérébral primitif ${ }^{10}$ et il semblerait qu'elles le soient aussi dans la lymphomatose cérébrale même si l'on manque de donnée ${ }^{11}$.

Le meilleur moyen de faire le diagnostic est évidemment l'examen anatomopathologique, cependant, en raison des localisations souvent profondes, une biopsie n'est parfois pas envisageable. Dans la très grande majorité des cas, il s'agit de lymphocytes $\mathrm{B}$ à grandes cellules de type «non-centre germinatif ». L'examen retrouve des cellules lymphocytaires avec de grands noyaux pléiomorphes et des nucléoles distincts infiltrant les structures corticales et sous-corticales, la substance blanche, les noyaux gris centraux et le thalamus, ainsi que le tronc cérébral et le cervelet ${ }^{12}$. Contrairement à la forme classique de lymphome cérébral primitif, il n'est que rarement retrouvé des plages de nécroses ${ }^{3}$. A noter, cependant, que l'aspect diffus de l'infiltration lymphoïde, à l'origine d'une faible densité de cellules malignes mais présentes sur de nombreuses coupes, rend l'interprétation anatomopathologique difficile, d'autant plus lorsque une corticothérapie a été instaurée auparavant comme chez notre patient réduisant d'autant plus la densité cellulaire (fig.6). 
La médiane de survie sur les 42 patients de la revue de C. Izquierdo était de 3 mois. Le traitement de référence consiste en de fortes doses intra-veineuses de méthotrexate $\left(3 \mathrm{~g} / \mathrm{m}^{2}\right)$ qui semble allonger significativement la survie de plusieurs mois avec même de rares cas de rémission ${ }^{13}, 14$. Il est intéressant de noter l'absence de réponse à la corticothérapie de notre patient ce qui n'est pourtant pas exceptionnel dans le cadre des lymphomes cérébraux primitifs puisque retrouvée dans plus de la moitié des cas ${ }^{15}$.

En conclusion, notre cas illustre le défi diagnostique ${ }^{14,16}$ posé par cette forme rare de lymphome cérébral primitif dont la présentation, notamment radiologique, est très différente de celle habituellement décrite dans la forme classique. Pourtant, ce diagnostic peut être évoqué par la combinaison des anomalies radiologique et du LCS. Il est d'autant plus important d'évoquer cette pathologie qu'elle évolue vers une issue fatale en quelques mois et qu'un traitement spécifique par méthotrexate pourrait améliorer significativement la survie des patients atteints.

\section{Liens d'intérêts}

Les auteurs déclarent ne pas avoir de liens d'intérêts. 


\section{Points essentiels}

- La lymphomatose cérébrale est une forme rare et diffuse de lymphome cérébral primitif.

- Son diagnostic est difficile et il doit être évoqué aussi chez un patient présentant une méningo-encéphalite subaigüe.

- A l'IRM cérébrale les anomalies sont, le plus souvent, des hypersignaux FLAIR supra et sous-tentorielles, symétriques, sans prise de contraste ni anomalie aux séquences de diffusion ou de perfusion. Cependant de nombreuses variantes de cette présentation classique sont possibles, notamment la présence de masse d'allure circonscrite et une prise de contraste.

- Le LCS peut-être d'une aide précieuse avec la présence d'une méningite lymphocytaire avec un taux d'IL10 élevé. On peut parfois y retrouver les lymphocytes anormaux monoclonaux.

- Le diagnostic définitif se fait à l'anatomopathologie, qui met en évidence de manière diffuse des cellules lymphomateuses isolées.

- Un diagnostic rapide est capital puisque qu'un traitement par méthotrexate à fortes doses semble bénéfique si celui-ci est instauré précocement. 
[1] Vezmar S, Becker A, Bode U, Jaehde U. Biochemical and clinical aspects of methotrexate neurotoxicity. Chemotherapy. 2003 May;49(1-2):92-104.

[2] Jain TK, Sharma P, Suman SK, Faizi NA, Bal C, Kumar R. Primary centralnervous system lymphoma with lymphomatosis cerebri in an immunocompetent child: MRI and 18FFDG PET-CT findings. Rev Esp Med Nucl Imagen Mol. 2013 Nov-Dec;32(6):394-6.

[3] Izquierdo C, Velasco R, Vidal N, Sánchez JJ, Argyriou AA, Besora S, et al.:

Lymphomatosis cerebri: a rare form of primary central nervous systemlymphoma. Analysis of 7 cases and systematic review of the literature. NeuroOncol. 2016 May;18(5):707-15.

[4] Lee PJ, Berrios I, Ionete C, Smith T. Lymphomatosis cerebri: diagnostic challenges and review of the literature. BMJ Case Rep. 2016 Nov 24;2016.

[5] Hatanpaa KJ, Fuda F, Koduru P, Young K, Lega B, Chen W. Lymphomatosis Cerebri:A Diagnostic Challenge. JAMA Neurol. 2015 Sep;72(9):1066-7.

[6] Ferreri AJ, Blay JY, Reni M, Pasini F, Gubkin A, et al. : Relevance of intraocular involvement in the management of primary central nervous system lymphomas. Ann Oncol. 2002 Apr;13(4):531-8.

[7] Raz E, Tinelli E, Antonelli M et al.: MRI findings in lymphomatosis cerebri: description of a case and revision of the literature. J Neuroimaging 2011 21:183-186.

[8] Sato H, Takahashi Y, Wada M, Shiono Y, Suzuki I, Kohno K, et al.: Lymphomatosis cerebri with intramedullary spinalcord involvement. Intern Med. 2013;52(22):2561-5.

[9] Sasayama T, Nakamizo S, Nishihara M, et al. Cerebrospinal fluid interleukin-10 is a potentially useful biomarker in immunocompetent primary central nervous system lymphoma (PCNSL). Neuro Oncol 14: 368-380, 2012

[10] Hashiguchi S, Momoo T, Murohashi Y, Endo M, Shimamura M, Kawasaki T, et al.: Interleukin 10 Level in the Cerebrospinal Fluid as a Possible Biomarker for Lymphomatosis Cerebri. Intern Med. 
[11] Kitai R, Hashimoto N, Yamate K, Ikawa M: Lymphomatosis cerebri: clinical characteristics, neuroimaging, and pathological findings. Brain Tumor Pathol2012 29:47-53.

[12] Samani A, Davagnanam I, Cockerell OC, Ramsay A, Patani R, Chataway J.:

Lymphomatosis cerebri: a treatable cause of rapidly progressive dementia. JNeurolNeurosurg Psychiatry. 2015 Feb;86(2):238-40.

[13] Watanabe M, Satoi H, Takahashi Y, Nishida N, Toda H, Matsumoto S.: Remission of lymphomatosis cerebri induced by corticosteroid and high-doses intravenous methotrexate. RinshoShinkeigaku. 2012;52(7):486-90.

[14] Murakami T, Yoshida K, Segawa M, Yoshihara A, Hoshi A, Nakamura K, et al.: A case of lymphomatosis cerebri mimicking inflammatory diseases. BMC Neurol. 2016 Aug $8 ; 16: 128$.

[15] Mathew BS, Carson KA, Grossman SA. : Initial response to glucocorticoids. Cancer. 2006 Jan 15;106(2):383-7.

[16] RiveroSanz E, TorralbaCabeza MÁ, Sanjuán Portugal F, García-Bragado F.: Lymphomatosis cerebri mimicking iatrogenic Creutzfeldt-Jakob disease. BMJ Case Rep. 2014 Sep 8;2014. 


\section{Légendes}

Figure 1 : Coupes de l'IRM cérébrale initiale en séquence T2 FLAIR. Les hypersignaux bipallidaux, bithalamiques, du mésencéphale, pédoncule cérébelleux moyen gauche et cortico-sous-cortical frontal droit sont mis en évidence par les flèches.

Figure 2 : Coupes de l'IRM cérébrale initiale en séquence T1 avec injection de gadolinium (A, B et C), en séquence de Diffusion (D et E) et en séquence de Perfusion (F).

Figure 3 : Coupes de l'IRM cérébrale à 4 mois du début des symptômes en séquence T2 FLAIR (A, B et C), en séquence T1 sans injection de gadolinium (D) et en séquence T1 avec injection de gadolinium (E et F).

Figure 4 : Coupes anatomopathologiques de la biopsie de la lésion de l'hémisphère cérébelleux gauche: A et $\mathrm{B}, 2$ sections consécutives avec une coloration par l'éosine et l'hématoxyline (A, 200X avec un encadré à 1000X) et un immunomarquage CD20 (B, 200X avec un encadré à 1000X). De grandes cellules atypiques isolées, rares, avec nucléoles proéminents et un noyau irrégulier étaient présentes dans le cortex cérébelleux (pointe de flèche dans A et l'encadré de A). Ces grandes cellules étaient CD20 immunopositives (CD20 +) (pointe de flèche en B et l'encadré dans B). C, immunomarquage CD20 (400X): un seul îlot de cellules CD20 + atypiques a été détecté dans toute la biopsie. Abréviations: gcl, granule cell layer; Ml, molecular layer.

Figure 5 : Coupe de l'IRM après première cure de méthotrexate en séquence T1.

Figure 6: Schéma comparatif de l'aspect histologique des lymphomes cérébraux primitifs, des lymphomatose cérébrale et chez notre patient. 


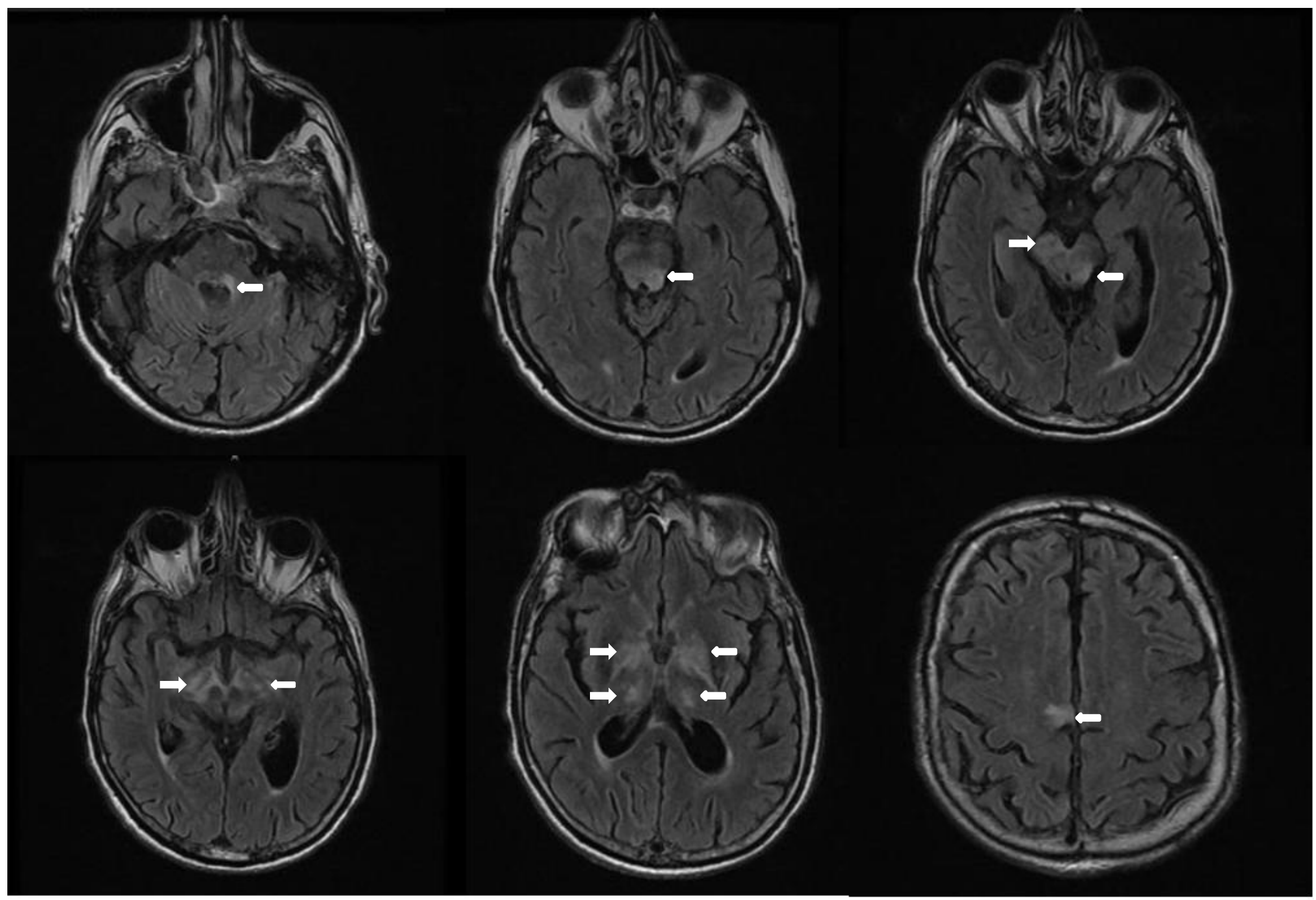




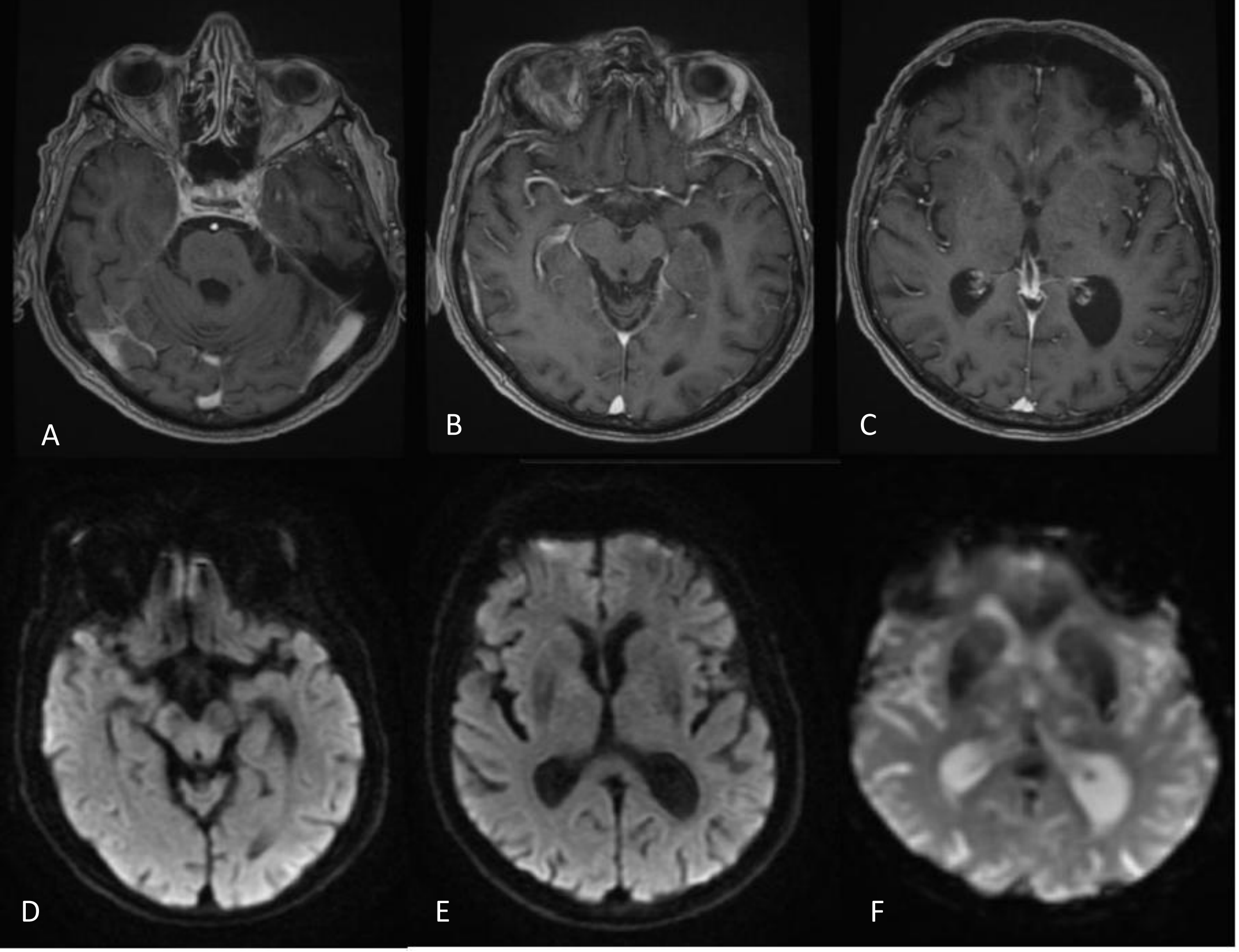


3
3
3
3

$(8)+1$

$(4 \times-5$

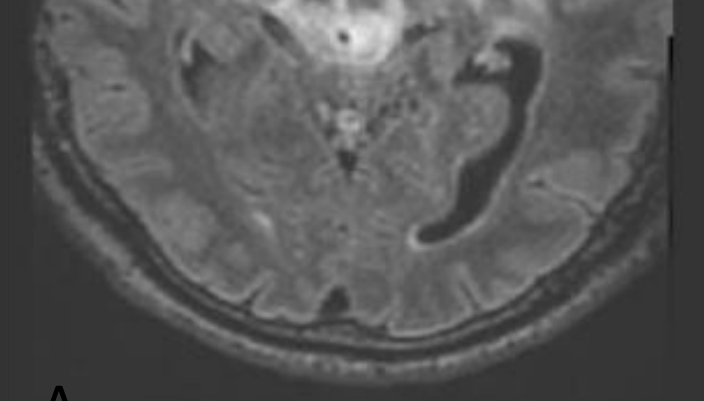

A
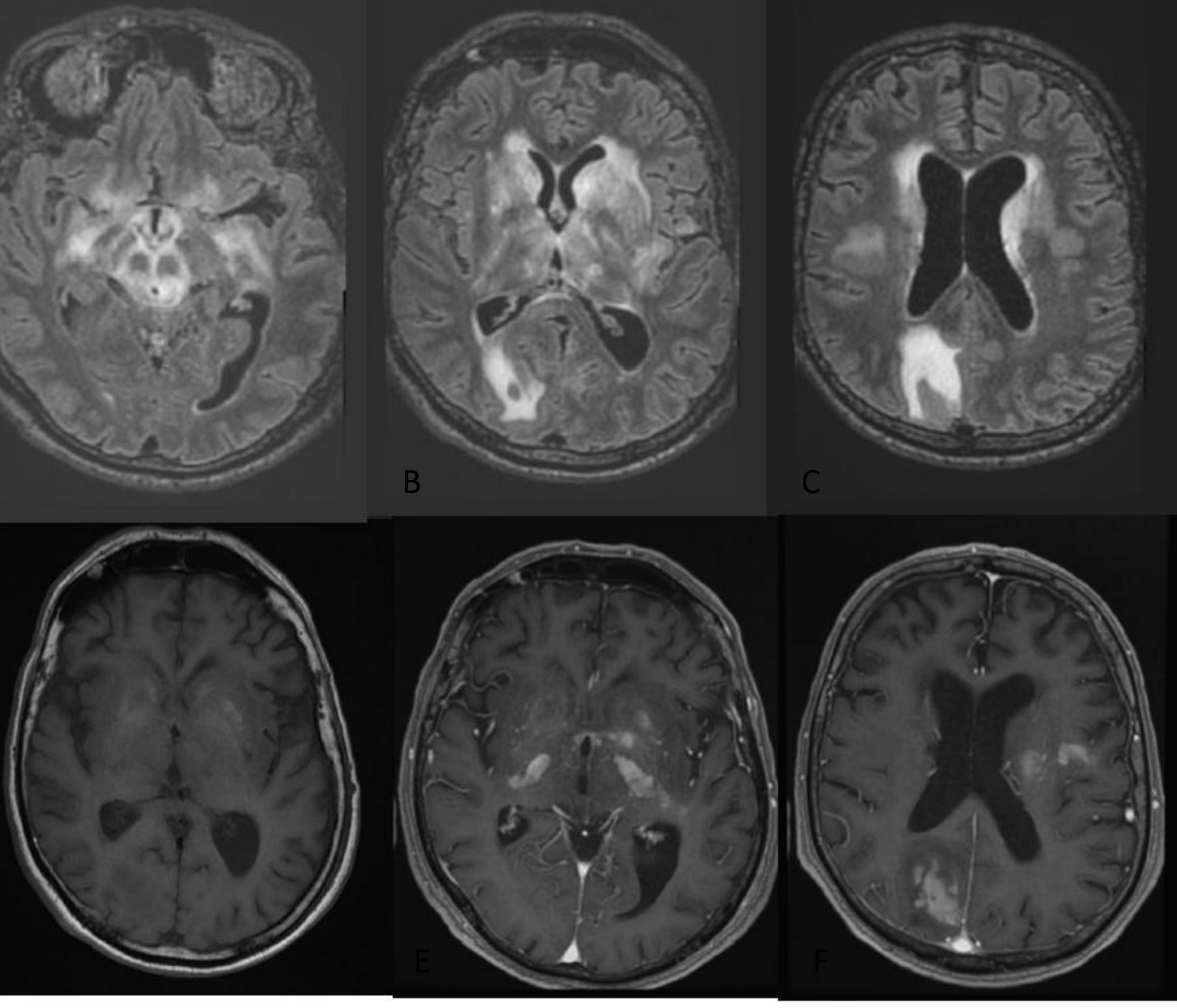


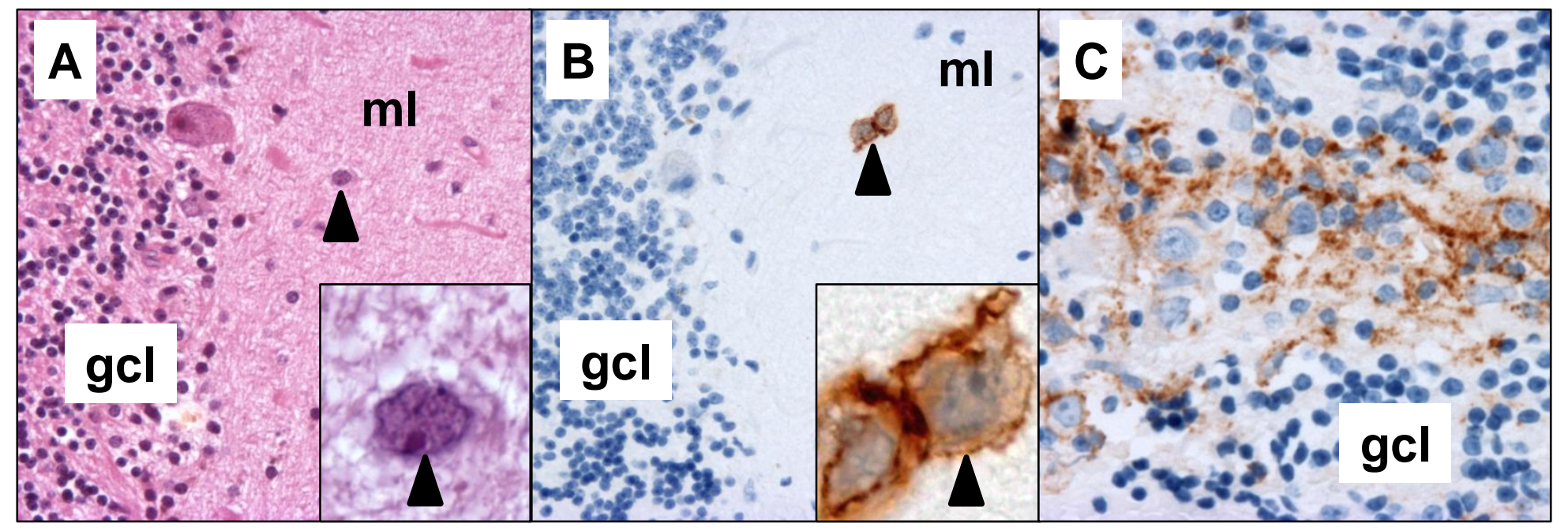




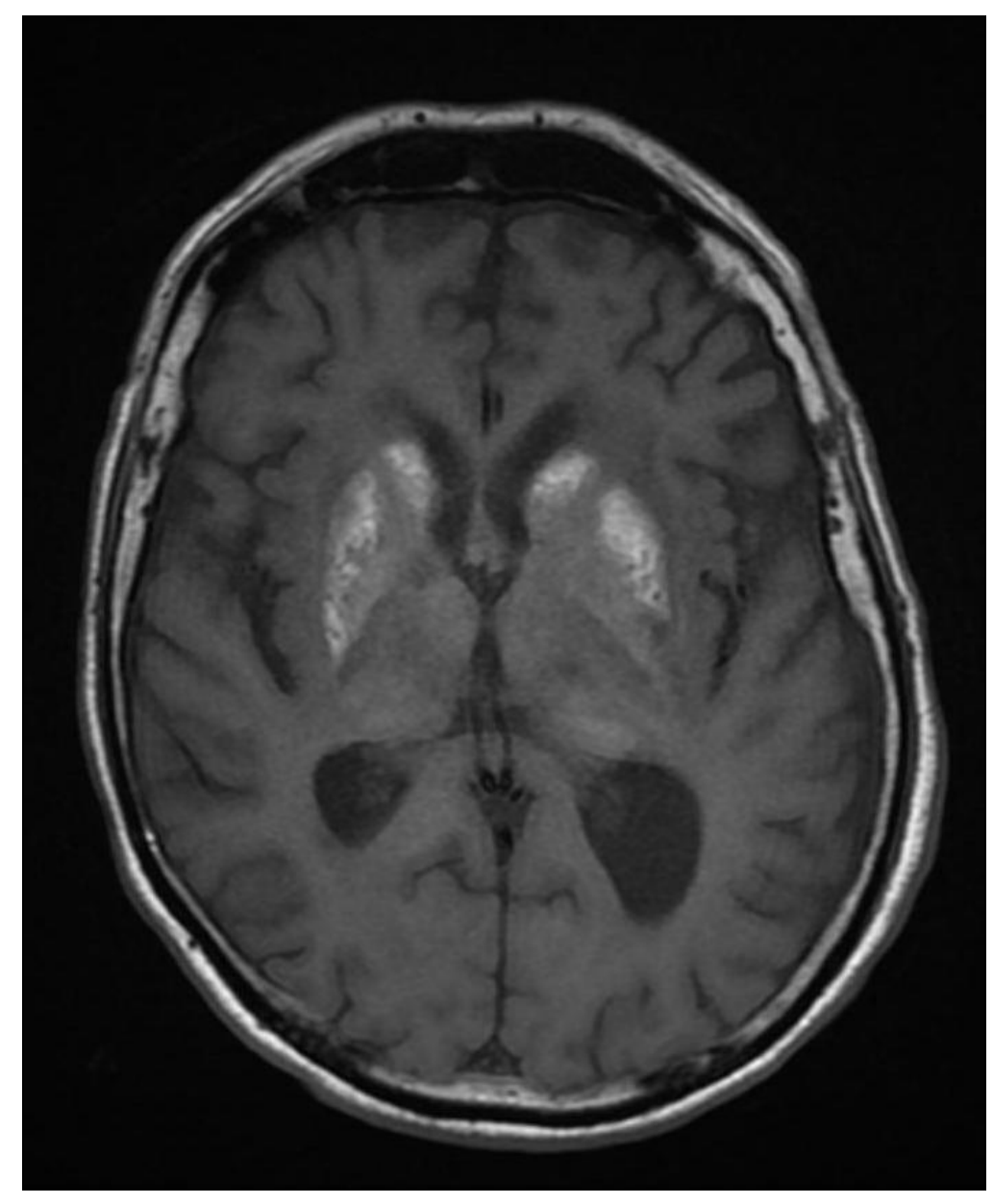


lymphome

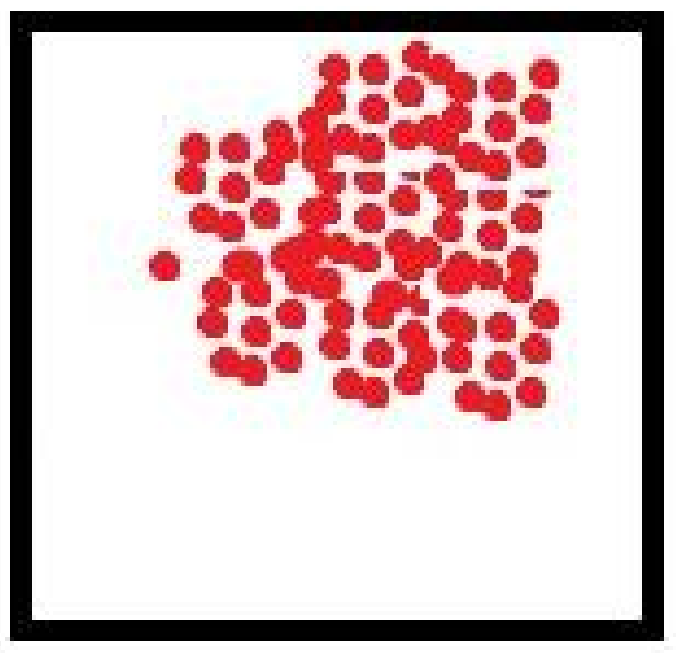

lymphomatosis

cerebri

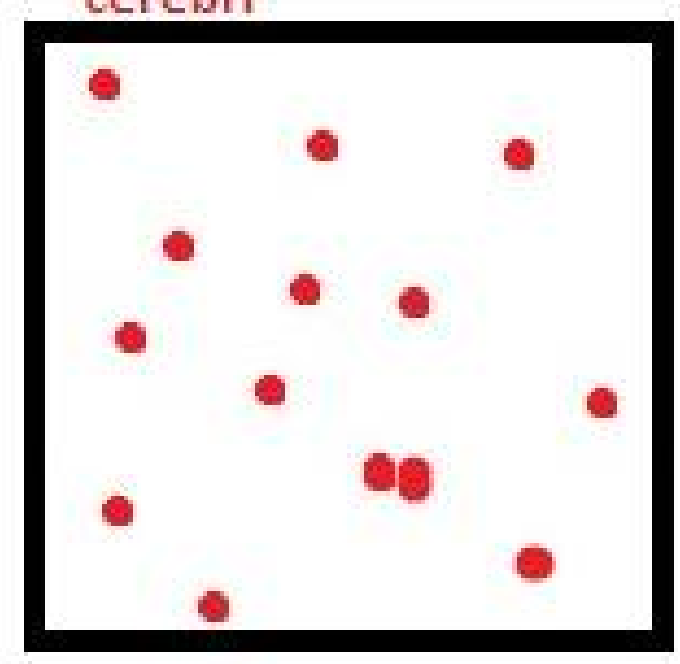

notre cas

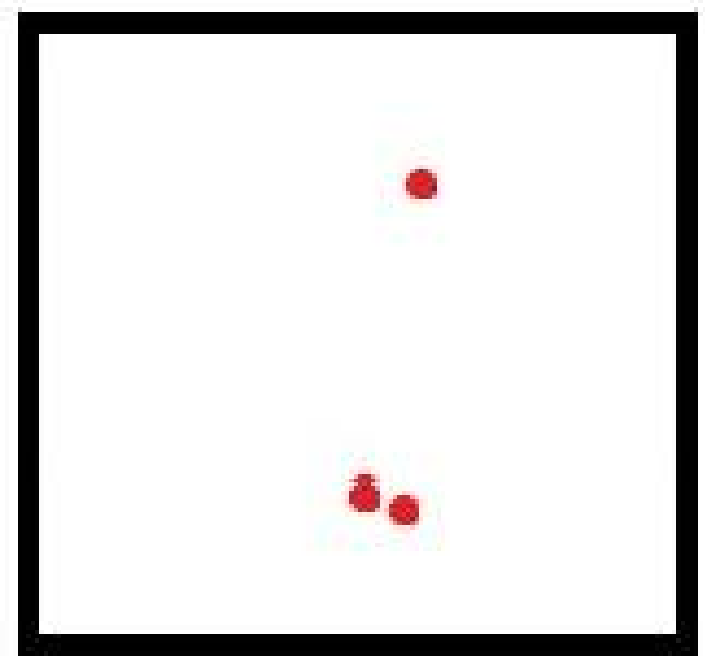

